\title{
2D/3D Deformable Registration Using a Hybrid Atlas
}

\author{
Thomas S.Y. Tang and Randy E. Ellis \\ School of Computing, Queen's University, Kingston, Canada K7L 3N6 \\ \{ttang, ellis\}@cs.queensu.ca
}

\begin{abstract}
Statistical atlases built by point distribution models (PDMs) using a novel hybrid 3D shape model were used for surface reconstruction. The hybrid shape model removes the need for global scaling in aligning training examples and instance generation, thereby allowing the PDM to capture a wider range of variations. The atlases can be used to reconstruct, or deformably register, the surface model of an object from just two to four 2D x-ray projections of the object. The methods was tested using proximal and distal femurs. Results of simulated projections and fluoroscopic images of cadaver knees show that the new instances can be registered with an accuracy of about $2 \mathrm{~mm}$.
\end{abstract}

\section{Introduction}

Variations of a shape in a population can be described using a statistical shape model (SSM). The point distribution model (PDM) proposed by Cootes et al. has been shown to be very successful in applications modeling anatomical objects.

In this paper, we propose a hybrid 3D shape model which can be used in a PDM framework. We show how to use the hybrid shape model and the resulting PDM to reconstruct the surface model of an object from a set of $2 \mathrm{D} x$-ray projections using intensity-based registration techniques.

Methods for reconstructing 3D surfaces by a PDM usually use the PDM in a simple way, requiring a global scale factor to align the training examples and to correct the surface model generated by a PDM. The problem of global scaling is that, because the scaling changes the original shapes, so the PDM is actually constructed from altered shapes. The use of the hybrid shape model removes the need of global scaling, therefore the PDM can capture a wider range of variations. Nonetheless, traditional PDM still has good variability given enough training examples are used. Fleute et al. [4] used a PDM for the reconstruction using the segmented contours of the $2 \mathrm{D}$ projections. Yao [7] used a PDM with tetrahedral meshes with intensity-based registration. Benameur et al. [1] used an edge-based method for registering 2D projections with the surface model generated by a PDM.

\section{Shape Modeling and Atlas Building}

A shape model should be able to describe both global (overall) characteristics and local (detail) characteristics of a shape. The hybrid shape model used in the present work explicitly modeled both characteristics. The global characteristics were described by a set 
of connecting inscribed spheres - in-spheres - contained within the bounding surface of the shape. The local characteristics were described by parameterizing a surface with respect to each in-sphere.

\subsection{Global Model}

The global model here, as is the case for Blum's medial-axis description [2], uses inspheres. One problem with medial-axis models, in general, is that a slight change in a shape can result in a very different set of medial axes. Instead of putting inscribed circles into a 2D shape (or in-spheres in 3D) that satisfy the medial constraints, one can instead fit in-spheres so that they take up the space inside the shape as much as possible. A crucial difference between the medial-axis description and the global model here is that spheres in the former may overlap, whereas in-spheres here serve as constraints on how subsequent in-spheres are positioned and sized. A given shape that is globally modeled by $k$ in-spheres can be parameterized by $k$ four-component tuples of the form $\left(\boldsymbol{c}_{i}, r_{i}\right)$, where $\boldsymbol{c}_{i}$ and $r_{i}$ are respectively the center and radius of the $i^{\text {th }}$ in-sphere. Figure 1 shows the global model of a proximal and distal femur.
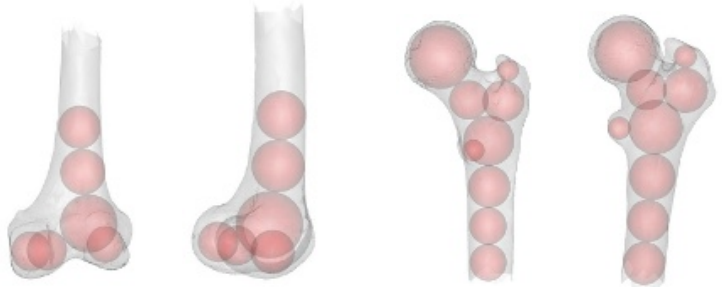

Fig. 1. Global model of the reference femur

\subsection{Local Model}

The local model parametrically represents the surface of a given shape around each in-sphere in the global model. In this work the parameterization was done by systematically shooting rays from the center of each sphere. Each ray had a parameter that represented the distance of the surface from the center of the sphere. Ideally the rays should be uniformly distributed on a sphere; this is only possible with the Platonic solids, but the rays would not be dense enough to capture the relevant details of the surface to be modeled. Nonetheless, approximation by a geodesic dome is sufficient. We used the octahedron and subdivided it such that it approximates a sphere with 1026 vertices and 2048 triangles. These vertices can then be used as directions of rays that emanate from the center.

Suppose that, for a given in-sphere, $n$ rays are produced. The local model of the shape's surface is derived from the intersections of the rays with the surface. This is done in two stages; first, the length of the rays are found and then a polygon is constructed from the tips of the rays. A set $\mathcal{L}$ consisting of $n$ distances suffice to parameterize the shape. 


\subsection{Merging the Global and Local Model}

If a given shape is represented by $k$ in-spheres, then it would be parameterized by $k$ tuples of the form $\left(\boldsymbol{c}_{i}, r_{i}, \mathcal{L}_{i}\right)$, where $\boldsymbol{c}_{i}$ and $r_{i}$ are the center and radius of the $i$-th sphere, and $\mathcal{L}_{i}$ is the $n$-dimensional set of ray lengths that describes the local shape. Each tuple can be used to reconstruct a mesh using the triangulation of the subdivided octahedron, in which the mesh describes part of the shape. The entire shape can be recovered by combining these meshes.

\subsection{Building a Shape Atlas}

Given a set of training examples, one of them, $\mathcal{M}_{\text {ref }}$ was selected as the reference example, and its shape parameter, $\boldsymbol{y}_{r e f}$, was determined. For a new training example $\mathcal{M}_{i}$, an affine transformation was performed with the reference example, such that $\mathcal{M}_{\text {ref }} \approx T_{\text {affine }}\left(T_{\text {rigid }}\left(\mathcal{M}_{i}\right)\right)$, where $T(\cdot)$ is a transformation. This affine transformation was used on the in-spheres locations of the reference example, so that they become the initial guess for the in-sphere location of the new training example. The in-sphere description is then determined in the same sequential order of the reference example. For the alignment, the only rigid part of the transformation is used. The local model of the new training example can be calculated after aligning it with the reference example, and the shape parameter $\boldsymbol{y}_{i}$ for $\mathcal{M}_{i}$ is now determined. Mathematically, $\mathcal{S}^{-1}\left(\boldsymbol{y}_{\text {ref }}\right) \approx T_{\text {affine }}\left(\mathcal{S}^{-1}\left(\boldsymbol{y}_{i}\right)\right)$.

With the shape parameters of all the training examples known, a hybrid statistical shape atlas was generated using principal component analysis (PCA) [3]. PCA reduces the dimension of the shape parameter from thousands to $\hat{y}$, whose dimension has only a few values, such that $\hat{y}=C(\boldsymbol{y})$, and $\boldsymbol{y} \approx C^{-1}(\hat{y})$.

The hybrid shape model provides a method for parameterizing a shape, which can be denoted as functions $\mathcal{S}(\cdot)$ and $\mathcal{S}^{-1}(\cdot)$, such that for a given a shape $\mathcal{M}$ that is represented as a triangle mesh:

$$
\begin{gathered}
\boldsymbol{y}=\left[\left(\boldsymbol{c}_{1}, r_{1}, \mathcal{L}_{1}\right), \ldots,\left(\boldsymbol{c}_{k}, r_{k}, \mathcal{L}_{k}\right)\right]^{T}=\mathcal{S}(\mathcal{M}) \\
\mathcal{M}^{\prime}=\mathcal{S}^{-1}(\boldsymbol{y})
\end{gathered}
$$

where $\mathcal{M}^{\prime}$ is a retriangulated version of $\mathcal{M}$.

\section{Shape Reconstruction}

Given data from a surface that is not one of the training examples, the parameters can be optimized to best match the given data, such that a model for this surface is reconstructed. This process also includes registration, because the given data is under some coordinate system that is different from the coordinate system of the atlas. Therefore the reconstruction gives the shape parameter $\hat{y}$, a rotation $R$ and translation $\boldsymbol{t}$, such that the mesh $\mathcal{S}\left(C^{-1}(\hat{y})\right)$ transformed by $T_{R, t}(\cdot)$ is the best match to the given data.

The given data may be of various forms. The two most important forms for orthopedic applications are when the data are 3D points obtained from the surface of an anatomical object, and when the data are 2D projections of an anatomical object. As for 
$2 \mathrm{D} / 3 \mathrm{D}$ rigid registration, a crucial component of reconstruction is the use of an appropriate similarity measure that can be used to compare the given data with an instance generated from the atlas. In other words, the reconstruction is essentially a minimization problem, that some error measure is minimized by using some values of $\hat{y} R$, and $\boldsymbol{t}$. Here we address the reconstruction using a few 2D projections.

Given a set of calibrated 2D projections (i.e., the projection geometry for each projection, and the relative poses of the projections are known) the reconstruction was done in an iterative manner in two steps. First, starting with the mean shape $(\hat{y}=\mathbf{0})$ and an initial estimate of the pose, an intensity-based registration [6] was performed so that a similarity measure [5] between the digital reconstructed radiographs (DRRs) of the shape, and the given projections, was optimized. By fixing the resulting transformation, the similarity measure was further optimized using the dimension-reduced shape parameter, which was done using a non-gradient-based optimization technique. By repeating these steps, the shape of the given surface was determined. Because we focus on orthopedic application, we used gradient correlation as the similarity measure. For the optimization of the shape parameter, we used the downhill simplex method.

Note that the shape atlas only provides a surface mesh, not an image volume that is required for DRR generation. To simulate an image volume using the surface mesh, one can intersect the mesh with a set of parallel planes. The result would be a contour of the shape, represented by line segments. Guided by the local surface normals, these segments were "grown" inward to simulate the thickness of the cortical bone in a real CT slice.

\section{Experiments}

The shape model was tested using 20 dry human femurs. CT scans were acquired for each femur, using an $(x, y)$ pixel size varying from 0.50 to $0.65 \mathrm{~mm}$ and a slice thickness of $1.25 \mathrm{~mm}$. The resolution of the CT volumes was $512 \times 512$, comprised from 380 to 400 slices. Left femurs were mirrored to produce only right-femur shapes. The surface meshes were computed from the CT volumes. The proximal and the distal femurs were treated separately.

In building and testing the atlas a leave-one-out approach was used, i.e., 19 femurs were used for training the atlas and the remaining one was used for testing the atlas. The dimensions of the shape parameters were about 6620 and 5180 for the proximal and distal femur; after performing PCA they were reduced to 9 and 7, with a cumulative variance of $85.9 \pm 0.4 \%$, and $86.1 \pm 0.5 \%$, respectively.

RMS error was used for quantifying the error in the reconstruction of a surface $\mathcal{M}$, which can be defined as the sum of the distances between all the mesh vertices of $\mathcal{M}$, and the reconstructed shape:

$$
R M S \operatorname{Error}(\hat{y}, R, \boldsymbol{t})=\sqrt{\sum_{\boldsymbol{m} \in \mathcal{M}} \| \boldsymbol{m}-\operatorname{closest}\left(\boldsymbol{m}, T_{R, \boldsymbol{t}}\left(\mathcal{S}\left(C^{-1}(\hat{y})\right)\right) \|^{2} /|\mathcal{M}|\right.}
$$

where $|\mathcal{M}|$ is the number of mesh vertices in $\mathcal{M}$. Note that a rigid transformation has already been performed for $\mathcal{M}$ in the simulation, so the RMS error contains not only the error in the shape, but also the error in the registration. 
Retrospective analyses show that the atlases were capable of describing the leftout femurs with mean RMS error \pm SD for the proximal and distal femur was $0.84 \pm$ $0.17 \mathrm{~mm}$ and $0.70 \pm 0.15 \mathrm{~mm}$ from $3 \mathrm{D}$ surface data.

\subsection{Reconstruction from 2D Projections}

Both simulated and cadaver data were used for testing.

For simulated data, reconstruction was done with two, three, and four images using all proximal and distal femurs. The view angle difference was approximately $15^{\circ}, 60^{\circ}$, and $90^{\circ}$ for reconstruction with two images, $15^{\circ}, 30^{\circ}$, and $45^{\circ}$ with three and four images. 2D projection images of the left-out femurs were simulated by DRRs with a resolution of $512 \times 512$. The mean initial pose error was normally distributed with zero mean and SD of $5^{\circ}$ and $10 \mathrm{~mm}$. Each set was done with and without calibration error. The calibration error included the error in finding the $\mathrm{x}$-ray source location of the fluoroscope (in-plane $\mathrm{SD}=0.25 \mathrm{~mm}$, out-of-plane $\mathrm{SD}=0.9 \mathrm{~mm}$ ), and the relative orientation between projections $\left(\mathrm{SD}=0.5^{\circ}, 0.2 \mathrm{~mm}\right)$.

Two human cadaver knees with soft tissues intact were used in this study. The distal femurs were implanted with seven fiducial markers, such that they could be used to provide ground truth for the pose of the femur from the fluoroscopic images (OEC 9800, General Electric, USA). The knees were flexed in nine or ten positions, with three images of about $45^{\circ}$ view angle difference for each position. CT scans with the same resolution of the dry bones were performed to determine the $3 \mathrm{D}$ models of the femurs, and the locations of the fiducial markers.

Table 1. Simulation of reconstruction using 2D projections for the proximal femur. All errors are in $\mathrm{mm}$. Note that the histograms contain cases that were failed.
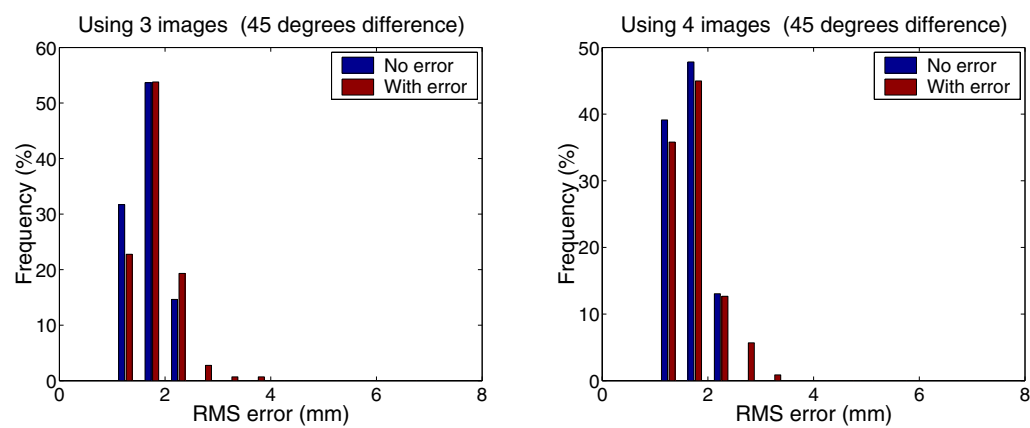

\begin{tabular}{|l||c|c|c||c|c|c||c|}
\hline \multirow{2}{*}{ Proximal Femur } & \multicolumn{3}{c||}{ No Error } & \multicolumn{3}{c|}{ With Error } & Number \\
& Mean & SD & Failure & Mean & SD & Failure & of cases \\
\hline \hline 2 images (all) & 1.80 & 0.45 & $0.9 \%$ & 2.03 & 0.54 & $2.9 \%$ & 490 \\
\hline 3 images (all) & 1.70 & 0.34 & $0.0 \%$ & 1.89 & 0.45 & $0.5 \%$ & 432 \\
\hline 4 images (all) & 1.66 & 0.34 & $0.0 \%$ & 1.76 & 0.45 & $0.0 \%$ & 512 \\
\hline 2 images, $90^{\circ}$ & 1.73 & 0.37 & $0.0 \%$ & 1.80 & 0.39 & $0.0 \%$ & 152 \\
\hline 3 images, $45^{\circ}$ & 1.66 & 0.32 & $0.0 \%$ & 1.79 & 0.38 & $0.0 \%$ & 145 \\
\hline 4 images, $45^{\circ}$ & 1.61 & 0.34 & $0.0 \%$ & 1.69 & 0.43 & $0.0 \%$ & 229 \\
\hline
\end{tabular}


Table 2. Simulation of reconstruction using 2D projections for the distal femur. All errors are in $\mathrm{mm}$. Note that the histograms contain cases that were failed.
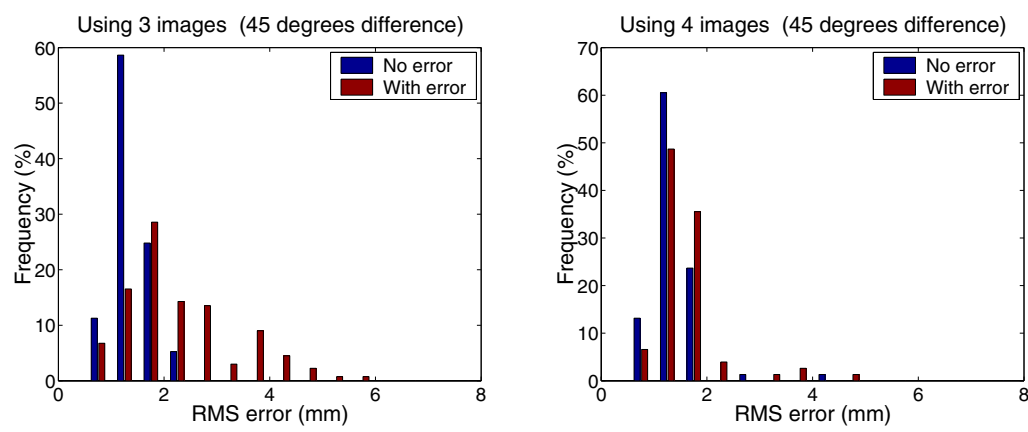

\begin{tabular}{|l||c|c|c||c|c|c||c|}
\hline \multicolumn{1}{|c||}{ Distal Femur } & \multicolumn{3}{c||}{ No Error } & \multicolumn{3}{c||}{ With Error } & Number \\
\cline { 2 - 7 } & Mean & SD & Failure & Mean & SD & Failure & of cases \\
\hline \hline 2 images (all) & 1.55 & 0.46 & $0.8 \%$ & 2.34 & 0.82 & $40.4 \%$ & 532 \\
\hline 3 images (all) & 1.46 & 0.40 & $0.2 \%$ & 1.99 & 0.70 & $15.0 \%$ & 513 \\
\hline 4 images (all) & 1.42 & 0.40 & $0.5 \%$ & 1.70 & 0.56 & $5.8 \%$ & 399 \\
\hline 2 images, $90^{\circ}$ & 1.42 & 0.39 & $0.8 \%$ & 2.20 & 0.82 & $44.4 \%$ & 133 \\
\hline 3 images, $45^{\circ}$ & 1.36 & 0.35 & $0.0 \%$ & 2.09 & 0.82 & $8.3 \%$ & 133 \\
\hline 4 images, $45^{\circ}$ & 1.31 & 0.34 & $1.3 \%$ & 1.57 & 0.50 & $1.3 \%$ & 76 \\
\hline
\end{tabular}

Table 3. Reconstruction from sets of three 2D projections of the distal femur of the two cadaver knees. Errors are in $\mathrm{mm}$.

\begin{tabular}{|l|c|c|c|c|}
\hline & Mean RMS Error & SD & $n$ & Failure \\
\hline Femur 1 & 1.95 & 0.55 & 171 & $6.9 \%$ \\
\hline Femur 2 & 1.72 & 0.25 & 190 & $0 \%$ \\
\hline
\end{tabular}

The average time taken for reconstruction with two, three, and four images were $4.5,6$, and 8 minutes. Table 1 and 2 summarize the overall simulation results, and also the setting which produced the best results ( 4 images at $45^{\circ}$ ). A mean RMS error of over $4 \mathrm{~mm}$ was considered a failure. As a reference, reconstruction of a femur phantom using 60 surface points had an mean RMS error of $1.35 \mathrm{~mm}$ for the proximal femur, and $1.34 \mathrm{~mm}$ for the distal femur.

\section{Discussion}

The error of reconstruction using 2D images were about $2 \mathrm{~mm}$, which is impressive because atlas-based reconstruction, or non-rigid registration, is a difficult problem. The use of more images significantly improved the accuracy. For both the proximal and distal femur, best results were obtained by using four images with view angles that were $45^{\circ}$ apart. The cadaver results were better than the simulation with errors, which means that the actual calibration errors were likely not as high as we simulated. 
Figures 2 and 3 show the average error for the proximal and distal femurs. In both cases, the error was extracted from a set of four test cases registered with four images at $45^{\circ}$ apart. These particular sets were chosen as they had a relatively high error, so they better illustrate the most problematic cases. In the proximal femur, the femoral head is the most stable, with very small errors except around the fovea capitis. The greater trochanter, the lesser trochanter, and the medial/posterior side were not accurately reconstructed. In the distal femur, most error occurred around the intercondylar notch. An explanation for the higher error in these regions is that they can only be seen in one viewing angles, and for some parts like the tip of the greater trochanter and the intercondylar notch, they are hardly visible, so they could only be inferred indirectly from the atlas simultaneously with other regions.

Although the atlases were capable of generating left-out femurs with a mean error of under $1 \mathrm{~mm}$, the error of the actual reconstruction was much higher. This does not imply that the deduced shape parameters are not optimal, as pose error is also included in the RMS error. Furthermore, the RMS errors reported here were only slightly higher than
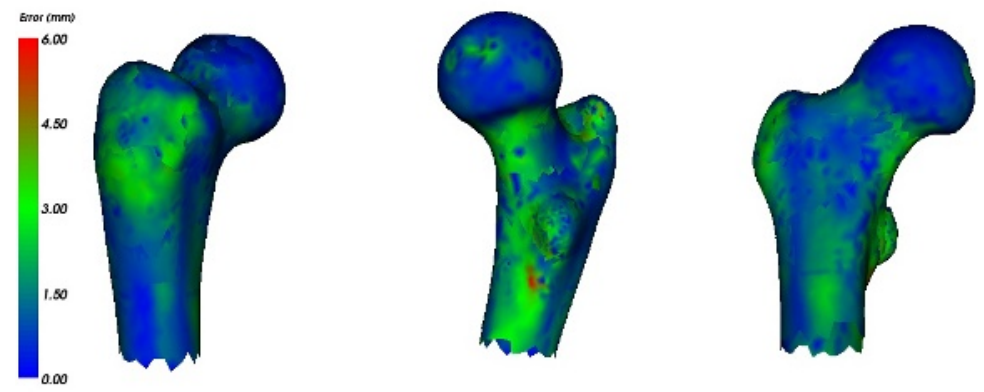

Fig. 2. Average error occurred in a proximal femur reconstructed by $2 \mathrm{D}$ images. The error was extracted from a set of four test cases registered with four images and $45^{\circ}$ apart, and with calibration error.
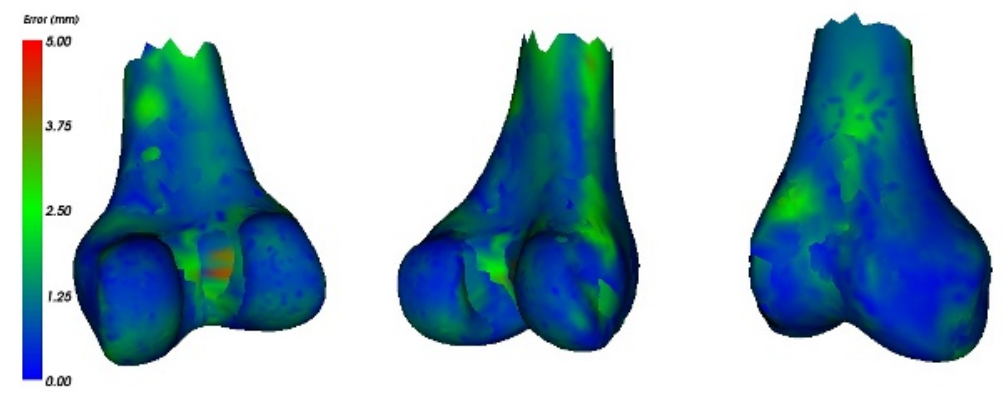

Fig. 3. Average error occurred in a distal femur reconstructed by $2 \mathrm{D}$ images. The error was extracted from a set of four test cases registered with four images and $45^{\circ}$ apart, and with calibration error. 
(if not comparable to) errors reported in the literature of intensity-based registration using the original CT scan.

In summary, we have shown that a PDM generated using our hybrid shape model can be used for registration using $2 \mathrm{D}$ x-ray projection with good accuracy, with the best results using four projections in $45^{\circ}$ difference. The only human intervention needed in the process of the reconstruction was the specification of the initial pose estimate.

\section{References}

1. S. Benameur, M. Mignotte, S. Parent, H. Labelle, W. Skalli, and J. E. Guise. 3D/2D registration and segmentation of scoliotic vertebrae using statistical models. Computerized Medical Imaging and Graphics, 27(5):321-337, 2003.

2. H. Blum and R. N. Nagel. Shape description using weighted symmetric axis features. Pattern Recognition, 10:167-180, 1978.

3. T. F. Cootes, C. J. Taylor, D. H. Cooper, and J. Graham. Active shape models - their training and application. Computer Vision and Image Understanding, 61(1):38-59, 1995.

4. M. Fleute, S. Lavallée, and R. Julliard. Incorporating a statistically based shape model into a system for computer-assisted anterior cruciate ligament surgery. Medical Image Analysis, 3(3):209-222, 1999.

5. G. P. Penney, J. Weese, J. A. Little, P. Desmedt, D. L. G. Hill, and D. J. Hawkes. A comparision of similarity measures for use in 2-D-3-D medical image registration. IEEE Transactions on Medical Imaging, 17(4):586-595, 1998.

6. T. S. Y. Tang, N. J. MacIntyre, H. S. Gill, R. A. Fellows, N. A. Hill, D. R. Wilson, and R. E. Ellis. Hardware-assisted 2D/3D intensity-based registration for assessing patellar tracking. In Medical Image Computing and Computer-Assisted Intervention - MICCAI 2004, volume 2, pages 1095-1096, 2004.

7. J. Yao. A Statistical Bone Density Atlas and Deformable Medical Image Registration. PhD thesis, Johns Hopkins University, 2001. 